\title{
ANCOVA versus Change Score for the Analysis of Nonexperimental Two-Wave Data:
}

\section{A Structural Modeling Perspective}

\author{
Oliver Lüdtke ${ }^{1,2}$ and Alexander Robitzsch ${ }^{1,2}$ \\ ${ }^{1}$ Leibniz Institute for Science and Mathematics Education, Kiel, Germany \\ ${ }^{2}$ Centre for International Student Assessment, Germany
}

Author Note

Correspondence concerning this article should be sent to Oliver Lüdtke, Leibniz Institute for Science and Mathematics Education (IPN), Olshausenstr. 62, 24118 Kiel, Germany. Email: oluedtke@leibniz-ipn.de 


\title{
ANCOVA VERSUS CHANGE SCORE
}

\begin{abstract}
There is an ongoing debate on whether the analysis of covariance (ANCOVA) or the change score approach is more appropriate when analyzing nonexperimental pre-post designs. In this article, we use a structural modeling perspective to clarify the different assumptions that are made by the ANCOVA and the change score approaches to identify the causal effect of a treatment variable. We show that the change score approach offers the option of controlling for unobserved confounders but relies on strong assumptions about the effects of these unobserved confounders and does not allow for dynamic causal relationships. By contrast, the ANCOVA approach is based on a selection-on-observables approach and assumes that all relevant confounders are measured. Furthermore, we illustrate conditions under which the two approaches give lower and upper bounds of the true treatment effect, and we discuss the role of measurement error. Implications for the analysis of nonexperimental two-wave data are discussed.
\end{abstract}

Keywords: causal effects, ANCOVA, change scores, longitudinal data, observational data 


\section{ANCOVA VERSUS CHANGE SCORE}

\section{ANCOVA versus Change Score for the Analysis of Nonexperimental Two-Wave Data: A Structural Modeling Perspective}

Assessing the effects of an intervention or construct on change in another construct is a central goal in virtually all areas of psychology. One popular strategy in nonexperimental research is to use a pre-post design in order to estimate how change in a construct is related to a particular treatment or construct (Little, 2013; Reichardt, 2019). For example, differential and developmental researchers are interested in how the occurrence of particular life events (e.g., job loss, marriage) affects changes in well-being or personality. In educational psychology, for example, researchers investigate how teacher characteristics (e.g., competence, motivation) predict changes in students' achievement. Most researchers rely on one of two basic strategies when analyzing the prospective effects of one construct on another construct in the context of two-wave data (Werts \& Linn, 1970). In the analysis of covariance (ANCOVA) or conditioning approach, the posttest $\left(Y_{2}\right)$ is conditioned on the pretest $\left(Y_{1}\right)$ by regressing the posttest on the pretest and the treatment variable $(T)$. In the change score approach, the difference between the Time 2 and Time 1 scores $\left(Y_{2}-Y_{1}\right)$ is regressed on the treatment variable $T$. As pointed out by many scholars, for a given application, it can be challenging to decide which of the two approaches is more appropriate (e.g., Köhler, Hartig, \& Schmid, 2020; Maris, 1998; O’Neill, Kreif, Grieve, Sutton, \& Sekhon, 2016; van Breukelen, 2013). However, the decision is often critical as the two approaches can yield results that substantially differ concerning the magnitude, sign, and statistical significance of the estimated treatment effect (Lord, 1967; Wright, 2006).

In the methodological literature there is an ongoing debate about whether the ANCOVA approach or the change score approach should be preferred for analyzing nonexperimental pre-post designs (e.g., Castro-Schilo \& Grimm, 2018; Eriksson \& Häggström, 2014; Farmus, Arpin-Cribbie, \& Cribbie, 2019; Gollwitzer, Christ, \& Lemmer, 2014; Stoolmiller \& Bank, 1995; Xiao, Higgins, \& Kasim, 2019). One frequently made 


\section{ANCOVA VERSUS CHANGE SCORE}

argument is that—from a descriptive perspective - the two approaches address different research questions. In the ANCOVA approach, for example, the focus is on whether the occurrence of a life event predicts well-being at Time 2 after controlling for the initial level in well-being at Time 1. By contrast, the change score approach estimates whether interindividual differences in change in well-being are associated with the occurrence of a life event. However, we suspect that psychological theories rarely provide researchers with enough guidance on how to differentiate between these two research approaches in a given application. In the present article, we take an alternative view and adopt a causal inference perspective on this issue (Glymour, Weuve, Berkman, Kawachi, \& Robins, 2005; Holland \& Rubin, 1983; Kenny, 1975; Kim \& Steiner, 2019; Maris, 1998; Steyer, 2005). Our main argument is that from a causal inference perspective, the two approaches address the same question (i.e., estimating the causal effect of the treatment) but rely on differentunfortunately untestable-assumptions about the effects of confounding variables.

More specifically, we use a structural modeling perspective (Pearl, Glymour, \& Jewell, 2016; see Rohrer, 2018, for a recent introduction, and Duncan, 1975, for an early treatment) to discuss the causal assumptions of the ANCOVA and change score approaches. We show that the ANCOVA approach is based on a selection-on-observables approach and assumes that all relevant confounder variables are measured and included in the regression model. By contrast, the change score approach offers the option of controlling for unobserved confounders but relies on strong assumptions about the effects of these unobserved confounders and does not allow for dynamic causal relationships (i.e., past outcomes affect current treatment). By clarifying the causal assumptions of these two widely used approaches, we hope to support researchers in utilizing the strength of longitudinal data and, at the same time, we hope to raise the awareness of the strong assumptions that are needed for making causal conclusions. This is also in line with recent prominent calls in psychology that nonexperimental research should begin to talk more openly about causal assumptions and 


\section{ANCOVA VERSUS CHANGE SCORE}

causal effects because causal questions are central to most psychological theories (e.g., Brick \& Bailey, 2020; Foster, 2010; Grosz, Rohrer, \& Thoemmes, 2020; Quynh Nguyen, Schmid, \& Stuart, 2020).

Before we start, we would like to point out that we are not concerned with the problem of measuring change at the individual level. Many different estimators have been proposed for quantifying change at the individual level (e.g., gain score, residualized gain), and there is a longstanding debate about which of these estimators provide the most accurate estimate of individual change (e.g., Lord, 1956; Cronbach \& Furby, 1970). As we hope to clarify, the accurate measurement of change at the individual level is distinct from the estimation of the treatment effect in a pre-post design (Campbell \& Kenny, 1999; Nicewander \& Price, 1978; for a comprehensive comparison of different estimators of individual change see Castellano \& McCaffrey, 2020).

\section{A Structural Model for Two-Wave Data}

In the following, we introduce a structural model for two-wave data that represents the causal relationships between the variables and allows us to clearly state the causal assumptions that are made by the two different approaches (Allison, 1990; Kenny, 1975; Kim \& Steiner, 2019). We assume that an outcome (e.g., well-being) is measured at two measurement waves and is denoted as $Y_{1}$ and $Y_{2}$. Our main goal is to estimate the effect of a treatment $T$ (e.g., the occurrence of a life event) on the outcome $Y_{2}$. Furthermore, we assume that a confounding variable $U$ (e.g., physical health) that affects both the outcomes $\left(Y_{1}\right.$ and $\left.Y_{2}\right)$ and the treatment is present (see also Figure 1):

$$
\begin{aligned}
& Y_{1}=\alpha_{1} U+\varepsilon_{1} \\
& T=\alpha_{T} U+\delta Y_{1}+\varepsilon_{2} \\
& Y_{2}=\alpha_{2} U+\beta Y_{1}+\gamma T+\varepsilon_{3} .
\end{aligned}
$$




\section{ANCOVA VERSUS CHANGE SCORE}

In the interests of simplicity and transparency, we assume that all effects are linear and that the variables are standardized (i.e., mean-centered with unit variance). We also assume that the variables are measured without error but, in a later section, we address how measurement error affects our conclusions.

\section{Figure 1}

Structural Model for Two-Wave Data: Effect of Treatment T on Outcome Y 2 With Effects of the Past Outcome $Y_{1}$ and a Confounder U. The Error Terms are not Depicted (see Equation 1)

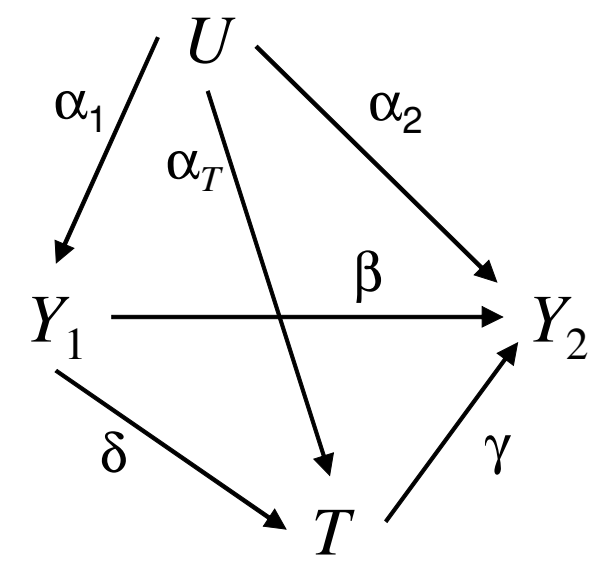

Four remarks about the data-generating model in Equation 1 seem to be in order. First, we assume that the treatment $T$ temporally precedes the outcome $Y_{2}$. In real applications, assumptions about the temporal ordering of treatment and outcome can be challenging with two-wave data. If $T$ is measured at the same time as $Y_{2}$, it is often difficult to rule out reverse causation as $Y_{2}$ could also have affected $T$. If a researcher is, for example, interested in the effect of motivation on achievement in a school subject (e.g., mathematics), and both motivation and achievement are assessed at Time 2, it needs to be ruled out that achievement has affected motivation (Marsh, Trautwein, Lüdtke, Köller, \& Baumert, 2005). If, instead, T and the past outcome $Y_{1}$ are assessed at the same time, it can be difficult to determine whether $Y_{1}$ is affected by $T$. In this case, $Y_{1}$ would not be a confounder but a mediator on the causal 


\section{ANCOVA VERSUS CHANGE SCORE}

pathway from $T$ to $Y_{2}$, and controlling for $Y_{1}$ would block some of the effect of $T$ on $Y_{2}$. Thus, in some settings, three waves of data would need to be available to distinguish between confounding and mediation effects. With three waves, it would be possible to establish temporal ordering by measuring the pretest at Time 1 , the treatment at Time 2 , and the outcome/posttest at Time 3 (for a further discussion see VanderWeele, Jackson, \& Li, 2016; VanderWeele, Mathur, \& Chen, 2018). Second, we are interested in the effect of the treatment at a single point in time. In many settings, the treatment also varies across time. For example, when assessing the effect of teachers' burnout on student achievement, teachers' burnout changes across time. With several waves of data, it would be possible to assess the effects of a treatment trajectory on final outcomes. However, the assumptions made when assessing the joint effect of a treatment over a series of time points are more complex (Hernán \& Robins, 2020; Robins, Hernán, \& Brumback, 2000; see also Daniel et al., 2013). Third, other datagenerating models could be proposed for two-wave data; there are many ways to address the issue of the estimation of treatment effects with longitudinal data. Later (see "Alternative Structural Model Using Transient and Stable Components" section), we show that an alternative data-generating model that has been suggested in the literature (Kenny, 1975) can be considered to be a special case of the model in Equation 1. Fourth, in order to simplify our discussion of the biases of the ANCOVA and change score approaches, we assume that the effects in the data-generating model are not negative, even though the formulas we provide also include conditions in which some of the effects are negative.

\section{Bias of ANCOVA and Change Score Estimators}

We now discuss the conditions under which the ANCOVA and change score approaches provide unbiased estimators of the treatment effect, under the assumption that the model in Equation 1 is true.

\section{ANCOVA Approach}




\section{ANCOVA VERSUS CHANGE SCORE}

For the ANCOVA approach, a regression model is specified that estimates the effect of the treatment $T$ on an outcome $Y_{2}$ measured at a later time point while controlling for the initial values of the outcome $Y_{1}$ :

$$
Y_{2}=b_{0}+\gamma_{\mathrm{ANCOVA}} T+b_{1} Y_{1}+\varepsilon
$$

where $b_{0}$ is the regression intercept and the ANCOVA estimator $\gamma_{\mathrm{ANCOVA}}$ is the regression coefficient of the regression of $Y_{2}$ on $T$ and $Y_{1}$. Thus, the ANCOVA approach controls for preexisting differences in the outcome when assessing the effect of the treatment. It can be considered to be a special case of a more general class of conditioning methods (e.g., matching methods) in which the causal effect is obtained by conditioning on the pretest and other observed covariates (see Morgan \& Winship, 2015). The basic idea is that the pretest is sufficient to control for the confounding effect of unmeasured covariates $U$. Notice that in the ANCOVA approach, the pretest has the same status as any other covariate. Under the assumption that the data-generating model in Equation 1 holds, the bias of the ANCOVA estimator is given by (see Appendix A):

$$
\gamma_{\mathrm{ANCOVA}}=\gamma+\frac{\alpha_{2} \alpha_{T}\left(1-\alpha_{1}^{2}\right)}{1-2 \delta \alpha_{1} \alpha_{T}-\delta^{2}-\alpha_{1}^{2} \alpha_{T}^{2}}
$$

The ANCOVA approach produces an unbiased estimate of the treatment effect if, conditional on $Y_{1}$, the unobserved covariate $U$ does not affect the treatment (i.e., $\alpha_{T}=0$ ) or the outcome $Y_{2}$ (i.e., $\alpha_{2}=0$ ). Another view of the ANCOVA approach is that it uses the past outcome and other observed covariates as a proxy for the unobserved confounder (Kim \& Steiner, 2019). It needs to be emphasized that the ANCOVA estimator is unbiased when the confounder $U$ is measured and included in the regression in Equation 2. Thus, the selection of covariates is crucial in the ANCOVA approach. Many scholars have pointed out that the pretest is the single most important covariate in many settings (e.g., Steiner, Cook, Shadish, \& Clark, 2010; VanderWeele, 2019).

\section{Change Score Approach}




\section{ANCOVA VERSUS CHANGE SCORE}

In the change score approach, the difference between the Time 2 and Time 1 scores is regressed on the treatment variable:

$$
Y_{2}-Y_{1}=b_{0}+\gamma_{\text {change }} T+\varepsilon
$$

where $\gamma_{\text {change }}$ indicates the difference in change in the outcome between the treatment and control groups. Even though the two approaches use the same variables in the regression model, three differences between the change score and ANCOVA approaches need to be emphasized. First, it is a necessary precondition for calculating the difference score that the measures at the two waves are operationally identical (i.e., $Y_{1}$ and $Y_{2}$ need to be in the same metric; Allison, 1990; Plewis, 1985). This is not the case in the ANCOVA approach because the pretest is treated like any other covariate. Second, when comparing the two approaches, it can be instructive to rewrite the regression of the change score approach as $Y_{2}=b_{0}+1 \cdot Y_{1}+$ $\gamma_{\text {change }} T+\varepsilon$ (by moving $Y_{1}$ from the left to the right side of the equation and fixing the regression coefficient to one). This expression emphasizes that the pretest receives a special status in the change score approach by restricting the effect of the pretest to one. It is vital to understand that this does not imply that the change score model is a restricted version of the ANCOVA model because our goal is not to maximize the explained variation in the regression but to control for confounding variables in the estimation of the treatment effect (Angrist \& Pischke, 2009). ${ }^{1}$ Third, it has been pointed out that both approaches imply different concepts of change and stability (e.g., Castro-Schilo \& Grimm, 2018; Maxwell \& Delaney, 2004; Newsom, 2015). In the ANCOVA approach, the way in which differences in $Y_{2}$ are associated with $T$ is assessed after individuals are equated on the initial values $Y_{1}$.

Therefore, this approach has sometimes also been referred to as the conditional change model or residualized change score model. By contrast, the change score approach takes the raw

\footnotetext{
${ }^{1}$ One may think of including $Y_{1}$ as an additional predictor in the change score approach. However, the treatment effect in the regression of $Y_{2}-Y_{1}$ on $Y_{1}$ and $T$ is equivalent to $\gamma_{\text {Ancova }}$ (Werts \& Linn, 1970; see also Kessler \& Greenberg, 1981).
} 


\section{ANCOVA VERSUS CHANGE SCORE}

individual change as the outcome variable and assesses how interindividual differences in change are associated with the treatment (unconditional change model). From this perspective, one could argue that the two approaches address different specific research questions and that determining which of the two approaches is more suitable depends on the research context.

However, from a causal inference perspective, we can derive the bias for the change score estimator under the assumption that the structural model in Equation 1 holds:

$$
\gamma_{\text {change }}=\gamma+\alpha_{T}\left(\alpha_{2}+\beta \alpha_{1}-\alpha_{1}\right)+\delta\left(\beta+\alpha_{1} \alpha_{2}-1\right)
$$

Thus, in the presence of an unobserved confounder $U$, two assumptions need to be met to ensure that the change score estimator is unbiased. First, the effect of the unobserved confounder $U$ needs to fulfill a very specific constraint, that is, $\alpha_{2}+\beta \alpha_{1}=\alpha_{1}$, so that the second term in Equation 5 is zero. This constraint essentially means that the effects of the confounder variable $U$ are stable across time. In the literature, this assumption is also known as the common trend assumption because it implies that, with a dichotomous treatment variable (treatment vs. control group), the unobservable trend of the outcome for the treatment group under the control condition is parallel to the observed trend for the control group (Lechner, 2010). Second, it needs to be assumed that the treatment is not affected by the past outcome $Y_{1}$ (i.e., $\delta=0$ ). This assumption has also been referred to as the assumption of "no dynamic causal relationships between the outcome and treatment variables" (Imai \& Kim, 2019, p. 477).

The main advantage of the change score approach is that it has the potential to control for the effect of unobserved confounder variables. This is attractive when researchers work with administrative data, which often only include standard demographic variables (e.g., age, sex, race, marital status) that have a limited potential to model the selection process for the treatment. However, the common trend assumption is very restrictive and often difficult to 


\section{ANCOVA VERSUS CHANGE SCORE}

defend (Angrist \& Pischke, 2009; Reichardt, 2019), even though the question of whether including covariates in the change score approach can increase the plausibility of the common trend assumption has been discussed in the literature (e.g., Lindner \& McConnell, 2019). We believe that the assumption of no dynamic causal relationships is even more crucial in many applications in psychological research as the occurrence or level of a current treatment often depends on previous levels of the outcome (e.g., the previous achievement affects the level of motivation, previous well-being affects the occurrence of a particular life event). In this case, it can be shown that even if the confounder $U$ is observed and included in Equation 2, the change score approach will, in general, produce biased estimates of the causal effect:

$$
\gamma_{\text {change }, U}=\gamma+\frac{\delta(1-\beta)\left(1-\alpha_{1}^{2}\right)}{1-2 \delta \alpha_{1} \alpha_{T}-\alpha_{T}^{2}-\delta^{2} \alpha_{1}^{2}} \text {. }
$$

As can be seen, when the previous outcome has an effect on the treatment $(\delta \neq 0$; causal dynamics), the change approach provides distorted estimates. Thus, in the change score approach there has to be a tradeoff between the possibility of controlling for the effects of unobserved confounders (with stable effects across time) and the possibility of taking causal dynamics into account (Imai \& Kim, 2019; Kim \& Steiner, 2019).

\section{Illustration: Bias of ANCOVA Versus Change Score Estimators}

Table 1 further illustrates the differences between the ANCOVA and change score estimators for different scenarios. We assumed that the true treatment effect would be modest in size (i.e., $\gamma=.20$ ) and that the outcome $Y$ would be moderately stable across time (i.e., $\beta=$ $.50)$, but we manipulated the effect of $U$ on $Y_{2}\left(\alpha_{2}=0, .1\right.$, and .2) and on the treatment $T\left(\alpha_{T}=\right.$ 0 and .3). We also varied whether the past outcome $Y_{1}$ had an effect on the treatment $(\delta=0$ and .3). As expected, the ANCOVA estimator tended to overestimate the true treatment effect and was unbiased under conditions in which $U$ did not have an effect on either $Y_{2}\left(\alpha_{2}=0\right)$ or the treatment $\left(\alpha_{T}=0\right)$. By contrast, the change score estimator was only unbiased if causal 


\section{ANCOVA VERSUS CHANGE SCORE}

dynamics were absent $(\delta=0)$ and either $U$ did not affect the treatment $\left(\alpha_{T}=0\right)$ or the common trend assumption was met $\left(\alpha_{2}=.2\right.$ so that $\left.\alpha_{2}+\beta \alpha_{1}=.2+.50 \times .40=.4=\alpha_{1}\right)$.

\section{Table 1}

Illustration of Bias in the ANCOVA and Change Score Estimators (see Figure 1): Size of the Estimated Treatment Effect as a Function of $\alpha_{2}, \delta$, and $\alpha_{T}$

\begin{tabular}{|c|c|c|c|c|}
\hline$\alpha_{2}$ & $\delta$ & $\alpha_{T}$ & $\gamma_{\text {ANCOVA }}$ & $\gamma_{\text {change }}$ \\
\hline 0 & 0 & 0 & .20 & .20 \\
\hline 0 & 0 & .3 & .20 & .14 \\
\hline 0 & .3 & 0 & .20 & .05 \\
\hline 0 & .3 & .3 & .20 & -.01 \\
\hline .1 & 0 & 0 & .20 & .20 \\
\hline .1 & 0 & .3 & .23 & .17 \\
\hline .1 & .3 & 0 & .20 & .06 \\
\hline .1 & .3 & .3 & .23 & .03 \\
\hline .2 & 0 & 0 & .20 & .20 \\
\hline .2 & 0 & .3 & .25 & .20 \\
\hline .2 & .3 & 0 & .20 & .07 \\
\hline .2 & .3 & .3 & .26 & .07 \\
\hline
\end{tabular}

Note. It is assumed that the true treatment effect is $\gamma=.20$, the stability of $Y$ is moderate $(\beta=$ $.50)$, and the effect of the confounder $U$ on $Y_{1}$ is $\alpha_{1}=.40$. Unbiased estimates are printed in bold.

Interestingly, the illustration reveals that the ANCOVA and change score estimators fulfill a useful bracketing relationship (Angrist \& Pischke, 2009; see also Ding \& Li, 2019). Namely, if the assumptions of the ANCOVA approach are met, then mistakenly applying the change score approach will underestimate the treatment effect. By contrast, if the assumptions of the change score approach hold, the ANCOVA approach will overestimate the treatment effect. It can be shown that under mild assumptions about the data-generating model in Equation 1, the ANCOVA estimator provides an upper bound and the change score estimator provides a lower bound for the true treatment effect:

$$
\gamma_{\text {change }} \leq \tau \leq \gamma_{\text {ANCOVA }}
$$




\section{ANCOVA VERSUS CHANGE SCORE}

More specifically, it needs to be assumed that the (cumulative) effect of $U$ on the outcome is smaller for the posttest than for the pretest, that is, $(1-\beta) \alpha_{1}-\alpha_{2}>0$, and that $\beta<1$ (see Appendix A; Angrist \& Pischke, 2009). The bracketing property is helpful in practice where one does not know if either the assumptions of the ANCOVA or the change score approach are true. Both approaches can be used to check the robustness of the results against different identifying assumptions for the treatment effect. The extent to which the two approaches converge often increases the credibility of the causal conclusions (Kenny, 1975; Reichardt, 2019).

\section{Alternative Structural Model Using Transient and Stable Components}

Our discussion of the conditions under which the ANCOVA and change score approaches give unbiased estimates of the treatment effect was based on a data-generating model that assumed a specific unobserved confounder variable $U$. However, other datagenerating processes for two-wave data are possible and have been proposed in the literature. Kenny (1975) introduced a model that decomposes potential confounding influences in terms of stable and transient components of the outcome variable. As can be seen in Figure 2, this model assumes that interindividual differences in the outcome and treatment are caused by a stable factor $U$ and transient factors $Z_{1}$ and $Z_{2}$. An interesting feature of this model is that it does not specify a direct effect of $Y_{1}$ on $Y_{2}$ but assumes that the stability in the outcome across time is due to the components $U, Z_{1}$, and $Z_{2}$. A very similar model was also used by Allison (1990; see Model 2) and Köhler et al. (2020) in their discussions of the ANCOVA and change score approaches. It should be emphasized that $U, Z_{1}$, and $Z_{2}$ are unmeasured, theoretical components that summarize the effects of stable and transient factors that influence $Y_{1}, T$, and $Y_{2}$.

Using this structural model, Kenny (1975; see also Allison, 1990) showed that the change score estimator is unbiased if the treatment assignment is caused by the stable component and not by the transient component (i.e., $\delta^{*}=0$ ), and if the effects of the stable 


\section{ANCOVA VERSUS CHANGE SCORE}

components are invariant across time $\left(\alpha_{1}^{*}=\alpha_{2}^{*}\right)$. By contrast, the estimation of the ANCOVA approach is unbiased when the treatment assignment is due to the transient component $Z_{1}$ but is not influenced by the stable component $\left(\alpha_{T}^{*}=0\right)$. However, Kenny's model (1975) can be seen as a special case of the structural model with an unobserved confounder $U$ in Equation 1 (see Appendix B). For example, in the case of the change score estimator, the assumption that treatment assignment is only caused by the stable component is equivalent to the assumption of no causal dynamics and common trends in the structural model with an unobserved confounder. We believe that in most applications, it is more convenient and more straightforward for researchers to conceptualize issues of confounding in terms of variables that are—at least in principle—measurable.

\section{Figure 2}

Alternative Structural Model That Decomposes the Effects of Unobserved Confounders Into Transient Components $Z_{1}$ and $Z_{2}$, and a Stable Component $U$ (Kenny, 1975; see also Allison, 1990). The Error Terms are not Depicted (see Equation B1)

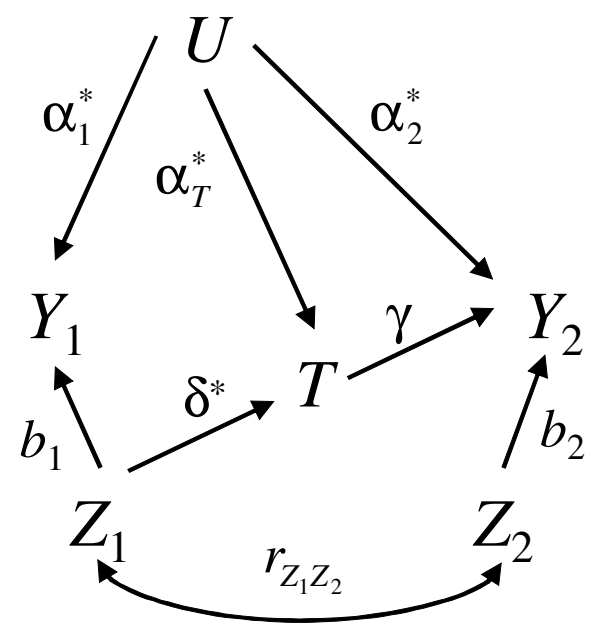

\section{The Role of Measurement Error}

In our discussion, we made the simplifying assumption that the variables are measured without error. In practical applications, the assessment of covariates, including the pretest, is often affected by measurement error. It has been pointed out that the presence of measurement 


\section{ANCOVA VERSUS CHANGE SCORE}

error can bias the estimates of the ANCOVA approach (Culpepper \& Aguinis, 2011;

Sengewald, Pohl, \& Steiner, 2019). Moreover, one advantage of the change score approach is that it is not sensitive to measurement error in the pretest (e.g., Kim \& Steiner, 2019). In the following, we extend the structural model in Equation 1, and we assume that we only have error-prone measures $\tilde{Y}_{1}, \widetilde{T}$, and $\tilde{Y}_{2}$ that are given by $\tilde{Y}_{1}=Y_{1}+E_{1}, \widetilde{T}=T+E_{2}$, and $\tilde{Y}_{2}=Y_{2}+$ $E_{3}$, where $E_{1}, E_{2}$, and $E_{3}$ are measurement errors with zero mean and variances $\sigma_{E_{1}}^{2}, \sigma_{E_{2}}^{2}$, and $\sigma_{E_{3}}^{2}$. The structural model with fallible measures is then represented by the following equations (see Figure 3$)^{2}$ :

$$
\begin{aligned}
& \tilde{Y}_{1}=Y_{1}+E_{1}=\alpha_{1} U+\varepsilon_{1}+E_{1} \\
& \tilde{T}=T+E_{2}=\alpha_{T} U+\delta Y_{1}+\varepsilon_{2}+E_{2} \\
& \tilde{Y}_{2}=Y_{2}+E_{3}=\alpha_{2} U+\beta Y_{1}+\gamma T+\varepsilon_{3}+E_{3} .
\end{aligned}
$$

In addition, we allow for the possibility that the measurement errors of the pretest and posttest are correlated, that is, $\rho_{E}=\operatorname{Cov}\left(E_{1}, E_{3}\right) /\left(\sigma_{E_{1}} \sigma_{E_{3}}\right)$. This is often a reasonable assumption when the same instrument is used as pretest and posttest and when the time lag between the two tests is not too long (Kim \& Steiner, 2019; Palmquist \& Green, 1992). As we assume that the true variables are standardized, the reliabilities of $\tilde{Y}_{1}, \widetilde{T}$, and $\tilde{Y}_{2}$ are given by $r_{1}=1 /\left(1+\sigma_{E_{1}}^{2}\right)$, $r_{2}=1 /\left(1+\sigma_{E_{2}}^{2}\right)$, and $r_{3}=1 /\left(1+\sigma_{E_{3}}^{2}\right)$.

To obtain consistent estimates of the treatment effect, an errors-in-variables approach (Carroll, Ruppert, Stefanski, \& Crainiceanu, 2006; Fuller, 1987; see also Culpepper \& Aguinis, 2011; Mayer, Dietzfelbinger, Rosseel, \& Steyer, 2016) can be used to adjust for measurement error in the observed variables. In this approach, the variance-covariance matrix of the observed predictor variables is corrected for the reliabilities. Note that the dependent

\footnotetext{
${ }^{2}$ Note that in the data-generating model, we assume that selection into the treatment depends on the true pretest $Y_{1}$. In certain designs, individuals are assigned to the treatment according to their observed values on the pretest (Reichardt, 2019). In this case, the observed pretest values should be included in the ANCOVA approach, and correcting for measurement error in $\tilde{Y}_{1}$ would result in biased estimates of the treatment effect (Sengewald et al., 2019).
} 


\section{ANCOVA VERSUS CHANGE SCORE}

variable can be measured with error because unreliability in the dependent variable does not result in biased estimates of unstandardized regression coefficients (Carroll et al., 2006). More specifically, the variances of the error-prone predictor variables are adjusted by their reliabilities, that is, $r_{1} \cdot \operatorname{Var}\left(\tilde{Y}_{1}\right)$ and $r_{2} \cdot \operatorname{Var}(\tilde{T})$. Furthermore, under the assumption that the errors are uncorrelated, the covariances between the observed variables provide consistent estimates of the covariances between the true variables (Carroll et al., 2006). Thus, the ANCOVA estimator adjusted for measurement error can be written as:

$\gamma_{\mathrm{ANCOVA}}^{*}=\frac{r_{1} \operatorname{Var}\left(\tilde{Y}_{1}\right) \operatorname{Cov}\left(\tilde{Y}_{2}, \tilde{T}\right)-\operatorname{Cov}\left(\tilde{Y}_{2}, \tilde{Y}_{1}\right) \operatorname{Cov}\left(\tilde{T}_{,} \tilde{Y}_{1}\right)}{r_{1} \operatorname{Var}\left(\tilde{Y}_{1}\right) r_{2} \operatorname{Var}(\tilde{T})-\operatorname{Cov}\left(\tilde{T}, \tilde{Y}_{1}\right)^{2}}$.

Note that it would also be possible to correct the covariances of the observed variables for correlated measurement error. However, as these correlations are difficult to determine, we assume that the presence of correlated measurement errors is ignored in the estimation of the treatment effect.

\section{Figure 3}

Structural Model for Two-Wave Data With Measurement Error: $\tilde{Y}_{1}, \tilde{T}$, and $\tilde{Y}_{2}$ are the Observed Measures That Assess $Y_{1}, T$, and $Y_{2}$ With Errors $E_{1}, E_{2}$, and $E_{3}$ (see Equation 8)

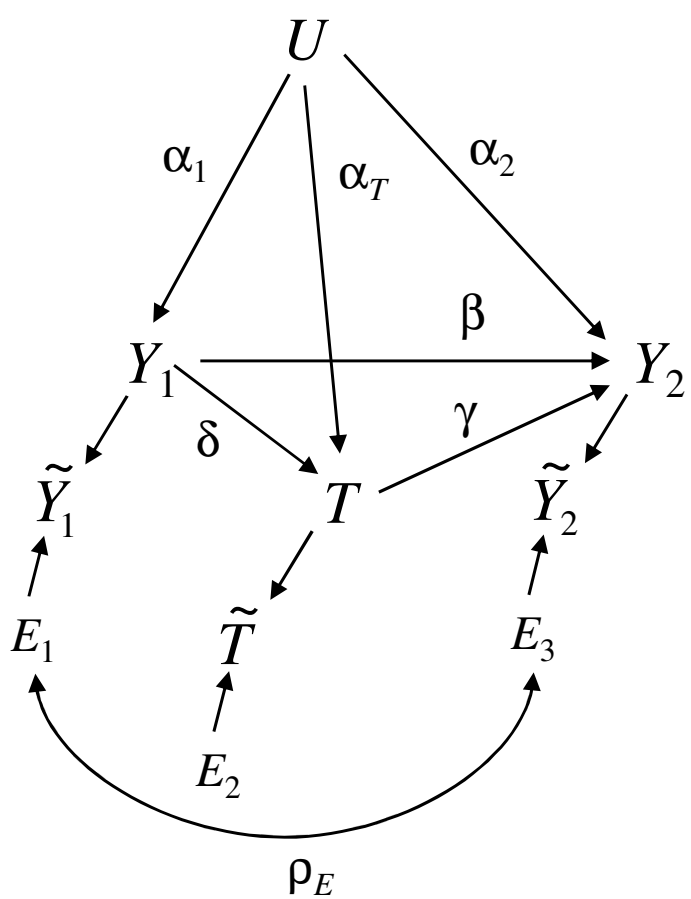




\section{ANCOVA VERSUS CHANGE SCORE}

The main challenge for the application of the errors-in-variables approach is that the reliabilities of the observed variables are required. Usually, the true reliabilities are not known, and researchers need to obtain estimates of the true reliabilities. Let $\lambda_{1}=\hat{r}_{1} / r_{1}$ and $\lambda_{2}$ $=\hat{r}_{2} / r_{2}$ denote the ratios of the estimated to the true reliabilities, indicating whether a researcher overestimates (i.e., $\lambda_{1}>1$ ) or underestimates (i.e., $\lambda_{1}<1$ ) the true reliability. Then the bias of the error-adjusted ANCOVA estimator can be derived as follows (see Appendix C):

$$
\gamma_{\mathrm{ANCOVA}}^{*}=\gamma+\frac{\alpha_{2} \alpha_{T}\left(\lambda_{1}-\alpha_{1}^{2}\right)+\left(\lambda_{1}-1\right)\left(\beta \delta+\delta \alpha_{1} \alpha_{2}+\beta \alpha_{1} \alpha_{T}\right)-\left(\lambda_{2}-1\right) \lambda_{1} \gamma-\rho_{E}\left(\alpha_{1} \alpha_{T}+\delta\right)}{\lambda_{1} \lambda_{2}-2 \delta \alpha_{1} \alpha_{T}-\delta^{2}-\alpha_{1}^{2} \alpha_{T}^{2}}
$$

The following observations can be made. First, if the reliabilities of the pretest and the treatment variables are correctly estimated $\left(\lambda_{1}=\lambda_{2}=1\right)$, and the errors are uncorrelated $\left(\rho_{E}=0\right)$, the bias expression for the unadjusted ANCOVA estimator is obtained (see

Equation 3). Second, mistakenly overestimating the reliability of the pretest $\left(\lambda_{1}>1\right)$, including the extreme case of ignoring measurement error in the pretest, will result, in general, in positively biased estimates of the treatment effect. In this case, the true pretest $Y_{1}$ acts as a confounder for the effect of $T$ on $Y_{2}$ (see the second term in the nominator of the fraction in Equation 10). Third, overestimating or ignoring the reliability of the treatment variable $\left(\lambda_{2}>1\right)$ will result in a downward bias on the absolute size of the treatment effect estimates. Interestingly, the size of this bias depends on the reliability of the pretest and is larger or smaller when the reliability of the pretest is overestimated or underestimated, respectively. Fourth, positively correlated errors of the pretest and posttest $\left(\rho_{E}>0\right)$ will introduce additional bias into the estimates of the treatment effect when the unobserved confounder $U$ affects the pretest and the treatment (i.e., $\alpha_{1} \neq 0$ and $\alpha_{T} \neq 0$ ) or the pretest has an effect on the treatment $(\delta \neq 0)$.

For the change score estimator, it is essential that the estimates of the treatment effect are not sensitive to measurement error in the pretest and posttest because measurement errors 


\section{ANCOVA VERSUS CHANGE SCORE}

in $\tilde{Y}_{1}$ and $\tilde{Y}_{2}$ do not affect estimates of $\operatorname{Cov}\left(\tilde{Y}_{2}-\tilde{Y}_{1}, \tilde{T}\right)$. However, the measurement error in $\tilde{T}$ needs to be taken into account and the corrected change score estimator $\gamma_{\text {change }}^{*}$ is calculated as $\operatorname{Cov}\left(\tilde{Y}_{2}-\tilde{Y}_{1}, \tilde{T}\right) /\left[r_{2} \operatorname{Var}(\tilde{T})\right]$. Under the assumption of the data-generating model in Equation 8 , the bias of $\gamma_{\text {change }}^{*}$ is given by:

$\gamma_{\text {change }}^{*}=\gamma+\frac{\lambda_{2}-1}{\lambda_{2}} \gamma+\frac{1}{\lambda_{2}}\left[\alpha_{T}\left(\alpha_{2}+\beta \alpha_{2}-\alpha_{1}\right)+\delta\left(\beta+\alpha_{1} \alpha_{2}-1\right)\right]$

Again, it can be seen that if the reliability of the treatment variable is correctly estimated $\left(\lambda_{2}=1\right)$, the bias expression for the unadjusted change score estimator is obtained (see Equation 5). Importantly, Equation 11 reveals that the adjusted change score estimator is not attenuated by correlated measurement errors. However, the change score estimator will still be biased if the common trend assumption is not met $\left(\alpha_{2}+\beta \alpha_{2} \neq \alpha_{1}\right)$ or if the pretest affects the treatment $(\delta \neq 0)$.

As pointed out, adjusting for error in the predictor variables requires estimates of the reliability to be available (Lockwood \& McCaffrey, 2014; Reichardt, 2019). In many applications, researchers use internal consistency measures of reliability (e.g., Cronbach's alpha), or they apply structural equation modeling (SEM; Bollen, 1989) with items as multiple indicators to correct for measurement error in the covariates. However, internal consistency measures of reliability only focus on measurement error that is caused by the finite number of items on the test. It has been argued that other sources of error (e.g., short-term fluctuations of the measure) should also be taken into account when controlling for the effects of error-prone variables (Kane, 2017; see also DeShon, 1998). A full discussion of different estimates of reliability is beyond the scope of the present article (see Brennan, 2001; Revelle \& Condon, 2019).

\section{Conclusion}

In the present article, we argue that from a causal perspective, the ANCOVA and change score approaches rely on different assumptions when identifying causal effects. We 


\section{ANCOVA VERSUS CHANGE SCORE}

emphasized that in a given application, both approaches can be wrong and that there is no statistical test that can tell researchers which of the two approaches is more plausible.

Furthermore, we showed that the change score approach offers the option of controlling for the effects of unobserved confounders. However, this comes at the price of a very restrictive assumption about the effects of the unobserved confounder (i.e., common trend assumption). This assumption often does not seem plausible in practice. Also, we showed that even if all relevant confounders are included in the change score approach, it does not allow for dynamic causal relationships and will produce biased estimates of the causal effect as long as the past outcome affects the current treatment. Given these limitations, we tend to prefer the ANCOVA approach for estimating treatment effects in pre-post designs because it provides a clear rationale for including observed covariates in the analysis (VanderWeele, 2019) ${ }^{3}$.

In practical applications of the ANCOVA approach, the selection of covariates and the correct specification of their effects (e.g., interactions and quadratic effects) is of central importance. Propensity score methods have been recommended as a flexible tool for adjusting for the effects of covariates when estimating treatment effects with nonexperimental data (e.g., Morgan \& Winship, 2015; Schafer \& Kang, 2008). In these methods, the propensity score (i.e., the conditional probability of treatment given the covariates) is used to balance the distribution of observed covariates between the treatment and control groups, in order to ensure that an estimated treatment effect is not due to differences in observed characteristics between the groups (Austin, 2011; Rosenbaum \& Rubin, 1983). A balance of the covariate distributions is achieved by matching, stratifying, or reweighting the observations according to the propensity score (Lunceford \& Davidian, 2004). However, it has been pointed out that

\footnotetext{
${ }^{3}$ In their discussion of the unconfoundedness-approach (equivalent to the ANCOVA approach in the present article) and the differences-in-differences approach (DID; equivalent to the change score approach), Imbens and Wooldridge (2009, see p. 70) conclude: "As a practical matter, the DID approach appears less attractive than the unconfoundedness-based approach in the context of panel data. It is difficult to see how making treated and control units comparable on lagged outcomes will make the causal interpretation of their difference less credible, as suggested by the DID assumptions."
} 


\section{ANCOVA VERSUS CHANGE SCORE}

the choice of a particular method of adjustment is not nearly as important as the set of measured covariates that is available for the adjustment (Reichardt, 2019; Steiner et al., 2010). Including a rich set of reliably measured covariates increases the credibility of the treatment effect estimates that are obtained from the ANCOVA approach. General recommendations for the selection of covariates are discussed by VanderWeele (2019). 


\section{ANCOVA VERSUS CHANGE SCORE}

\section{References}

Allison, P. (1990). Change scores as dependent variables in regression analysis. Sociological Methodology, 20, 93-114.

Angrist, J. D., \& Pischke, J.-S. (2009). Mostly harmless econometrics: An empiricist's companion. Princeton, NJ: Princeton University Press.

Austin, P. C. (2011). An introduction to propensity score methods for reducing the effects of confounding in observational studies. Multivariate Behavioral Research, 46, 399-424.

Brennan, R. L. (2001). Generalizability theory. New York: Springer.

Brick, T. R. \& Bailey, D. H. (2020). Rock the MIC: The matrix of implied causation, a tool for experimental design and model checking. Advances in Methods and Practice in Psychological Science. Advance online publication. doi: 10.1177/2515245920922775

Bollen, K. (1989). Structural equations with latent variables. New York, NY: John Wiley.

Campbell, D. T., \& Kenny, D. A. (1999). A primer on regression artifacts. New York:

Guilford Press.

Carroll, R. J., Ruppert, D., Stefanski, A., \& Crainiceanu, C. (2006). Measurement error in nonlinear models: A modern perspect ive. London: Chapman \& Hall/CRC.

Castellano, K. E., \& McCaffrey, D. F. (2020). Comparing the accuracy of student growth measures. Journal of Educational Measurement, 57, 71-91.

Castro-Schilo, L., \& Grimm, K. J. (2018). Using residualized change versus difference scores for longitudinal research. Journal of Social and Personal Relationships, 35, 32-58.

Cronbach, L. J., Furby, L. (1970). How should we measure "change"—or should we? Psychological Bulletin, 74, 68-80.

Culpepper, S. A., \& Aguinis, H. (2011). Using analysis of covariance (ANCOVA) with fallible covariates. Psychological Methods, 16, 166-178. 


\section{ANCOVA VERSUS CHANGE SCORE}

Daniel, R.M., Cousens, S. N., De Stavola, B. L., Kenward, M. G., \& Sterne, J. A. C. (2013). Methods for dealing with time-dependent confounding. Statistics in Medicine, 32, $1584-1618$.

DeShon, R. P. (1998). A cautionary note on measurement error corrections in structural equation models. Psychological Methods, 3, 412-423.

Ding, P., \& Li, F. (2019). A bracketing relationship between difference-in-differences and lagged-dependent-variable adjustment. Political Analysis, 27, 605-615.

Duncan, O. D. (1975). Introduction to structural equation models. New York: Academic Press.

Eriksson K., \& Häggström, O. (2014). Lord's paradox in a continuous setting and a regression artifact in numerical cognition research. PLoS ONE 9(4): e95949.

Farmus, L. Arpin-Cribbie, C. A., \& Cribbie, R. A. (2019). Continuous predictors of pretestposttest change: Highlighting the impact of the regression artifact. Frontiers of Applied Mathematics and Statistics, 4, 64.

Foster, E. M. Causal inference and developmental psychology. Developmental Psychology, $46,1454-1480$.

Fuller, W. (1987). Measurement error models. New York, NY: John Wiley.

Glymour, M. M., Weuve, J., Berkman, L. F., Kawachi, I., \& Robins, J. M. (2005). When is baseline adjustment useful in analyses of change? An example with education and cognitive change. American Journal of Epidemiology, 162, 267-278.

Gollwitzer, M., Christ, O., \& Lemmer, G. (2014). Individual differences make a difference: On the use and the psychometric properties of difference scores in social psychology. European Journal of Social Psychology, 44, 673-682.

Grosz, M. P., Rohrer, J. M., \& Thoemmes, F. (2020). The taboo against explicit causal inference in non-experimental psychology. Perspectives on Psychological Science. Advance online publication. doi: 10.1177/1745691620921521 


\section{ANCOVA VERSUS CHANGE SCORE}

Hernán, M . A., \& Robins, J. M. (2020). Causal inference: What if. Boca Raton, FL: Chapman \& Hall/CRC.

Holland, P. W., \& Rubin, D. B. (1983). On Lord's Paradox. In H. Wainer \& S. Messick (Eds.), Principals of modern psychological measurement (pp. 3-25). Hillsdale: Erlbaum.

Imai, K., \& Kim, I. S. (2019). When should we use fixed effects regression models for causal inference with longitudinal data? American Journal of Political Science, 63, 467-490.

Imbens, G. W., \& Wooldridge, J. M. (2009). Recent developments in the econometrics of program evaluation. Journal of Economic Literature, 47, 5-86.

Kane, M. T. (2017). Measurement error and bias in value-added models (Research Report No. RR-17-25). Princeton, NJ: Educational Testing Service.

Kenny, D. A. (1975). A quasi-experimental approach to assessing treatment effects in the nonequivalent control group design. Psychological Bulletin, 82, 345-362.

Kessler, R. C., \& Greenberg, D. F. (1981). Linear panel analysis: Models of quantitative change. New York: Academic Press.

Kim, Y., \& Steiner, P. M. (2019). Gain scores revisited: A graphical modeling perspective. Sociological Methods and Research. Advance online publication. doi: $10.1177 / 0049124119826155$

Köhler, C., Hartig, J., \& Schmid, C. (2020). Deciding between the covariance analytical approach and the change-score approach. Multivariate Behavioral Research. Advance online publication. doi: 10.1080/00273171.2020.1726723

Lechner, M. (2010). The estimation of causal effects by difference-in-difference methods. Foundations and Trends in Econometrics, 4, 165-224.

Lindner, S. L., \& McConnell, K. J. (2019). Difference-in-differences and matching on outcomes: A tale of two unobservables. Health Service and Outcomes Research Methodology, 19, 127-144. 


\section{ANCOVA VERSUS CHANGE SCORE}

Little, T. D. (2013). Longitudinal structural equation modeling. New York, NY: Guilford Press.

Lockwood, J. R., \& McCaffrey, D. F. (2014). Correcting for test score measurement error in ANCOVA models for estimating treatment effects. Journal of Educational and Behavioral Statistics, 39, 22-52.

Lord, F. M. (1956). The measurement of growth. Educational and Psychological Measurement, 16, 421-437.

Lord, F. M. (1967). A paradox in the interpretation of group comparisons. Psychological Bulletin, 68, 304-305.

Lunceford, J. K., \& Davidian, M. (2004). Stratification and weighting via the propensity score in estimation of causal treatment effects: a comparative study. Statistics in Medicine, 23, 2937-2960.

Maris, E. (1998). Covariance adjustment versus gain scores—revisited. Psychological Methods, 3, 309-327.

Marsh, H.W., Trautwein, U., Lüdtke, O., Köller, O., \& Baumert, J. (2005). Academic selfconcept, interest, grades, and standardized test scores: Reciprocal effects models of causal ordering. Child Development, 76, 397-416.

Mayer, A., Dietzfelbinger, L., Rosseel, Y., \& Steyer, R. (2016). The EffectLiteR approach for analyzing average and conditional effects. Multivariate Behavioral Research, 51, 374391.

Maxwell, S. E., \& Delaney, H. D. (2004). Designing experiments and analyzing data: A model comparison perspective. Mahwah, NJ: Erlbaum.

Morgan, S. L., \& Winship, C. (2015). Counterfactuals and causal inference: Methods and principles for social research (2nd ed.). Cambridge: University Press.

Newsom, J. T. (2015). Longitudinal structural equation modeling: A comprehensive introduction. New York: Routledge. 


\section{ANCOVA VERSUS CHANGE SCORE}

Nicewander, W. A., \& Price, J. M. (1978). Dependent variable reliability and the power of significance tests. Psychological Bulletin, 85, 405-409.

O’Neill, S. O., Kreif, N., Grieve, R., Sutton, M., \& Sekhon, J. S. (2016). Estimating causal effects: Considering three alternatives to difference-in-difference estimation. Health Service and Outcomes Research Methodology, 16, 1-21.

Palmquist, B., \& Green, D. P. (1992). Estimation of models with correlated measurement errors from panel data. Sociological Methodology, 22, 119-146.

Pearl, J., Glymour, M., \& Jewell, N. P. (2016). Causal inference in statistics. A primer. Chichester, UK: Wiley.

Plewis, I. (1985). Analysing change: Methods for the measurement and explanation of change in the social sciences. Chichester, UK: Wiley.

Quynh Nguyen, T., Schmid, I., \& Stuart, E. (2020). Clarifying causal mediation analysis for the applied researcher: Defining effects based on what we want to learn. Psychological Methods.

Reichardt, C. S. (2019). Quasi-Experimentation: A guide to design and analysis. New York, NY: Guilford Press.

Revelle, W., \& Condon, D. M. (2019). Reliability from $\alpha$ to $\omega$ : A tutorial. Psychological Assessment, 31, 1395-1411.

Robins, J. M., Hernán, M. A., \& Brumback, B. (2000). Marginal structural models and causal inference in epidemiology. Epidemiology, 11, 550-560.

Rohrer, J. M. (2018). Thinking clearly about correlations and causation: Graphical causal models for observational data. Advances in Methods and Practices in Psychological Science, 1, 27-42.

Rosenbaum P. R., \& Rubin D. B. (1983). The central role of the propensity score in observational studies for causal effects. Biometrika, 70, 41-55. 


\section{ANCOVA VERSUS CHANGE SCORE}

Schafer, J. L., \& Kang, J. (2008). Average causal effects from nonrandomized studies: A practical guide and simulated example. Psychological Methods, 13, 279-313.

Sengewald, M.-A., Pohl, S., \& Steiner, P. M. (2019). When does measurement error in covariates impact causal effect estimates? - Analytical derivations of different scenarios and an empirical illustration. British Journal of Mathematical and Statistical Psychology, 72, 244-270.

Steiner, P. M., Cook, T. D., Shadish, W. R., \& Clark, M. H. (2010). The importance of covariate selection in controlling for selection bias in observational studies. Psychological Methods, 15, 250-267.

Steyer, R. (2005). Analyzing individual and average causal effects via structural equation models. Methodology, 1, 39-54.

Stoolmiller, M., \& Bank, L. (1995). Autoregressive effects in structural equation models: We see some problems. In J. M. Gottman (Ed.), The analysis of change (pp. 261-276). Mahwah, NJ: Erlbaum.

Van Breuckelen, G. J. P. (2013). ANCOVA versus CHANGE from baseline in nonrandomized studies: The difference. Multivariate Behavioral Research, 48, 895922.

VanderWeele, T. J. (2019). Principles of confounder selection. European Journal of Epidemiology, 34, 211-219.

VanderWeele, T. J., Jackson, J. W., \& Li, S. (2016). Causal inference and longitudinal data: A case study of religion and mental health. Social Psychiatry and Psychiatric Epidemiology, 51, 457-1466.

VanderWeele, T. J., Mathur, M. B., \& Chen, Y. (2018). Outcome-wide longitudinal designs for causal inference: A new template for empirical studies. arXiv, arXiv:1810.10164

Werts, C. E., \& Linn, R. L. (1970). A general linear model for studying growth. Psychological Bulletin, 73, 17-22. 


\section{ANCOVA VERSUS CHANGE SCORE}

Wright, D. B. (2006). Comparing groups in a before-after design: When t test and ANCOVA produce different results. British Journal of Educational Psychology, 76, 663-675.

Xiao, Z. M., Higgins, A., \& Kasim, A. (2019). An empirical unraveling of Lord's Paradox. The Journal of Experimental Education, 87, 17-32. 


\section{ANCOVA VERSUS CHANGE SCORE}

\section{Appendix A: Derivations of Bias for the ANCOVA and Change Score Approaches}

In this appendix, we provide bias derivations for the ANCOVA and change score approaches. All variables are assumed to be standardized. The covariances between the observed variables $Y_{1}, Y_{2}$, and $T$ are derived as follows: $\operatorname{Cov}\left(T, Y_{1}\right)=\delta+\alpha_{1} \alpha_{T}, \operatorname{Cov}\left(Y_{2}, Y_{1}\right)=$ $\beta+\delta \gamma+\alpha_{1} \alpha_{2}+\alpha_{1} \alpha T \gamma ; \operatorname{Cov}\left(Y_{2}, T\right)=\gamma+\beta \delta+\alpha_{2} \alpha_{T}+\beta \alpha_{1} \alpha_{T}+\delta \alpha_{1} \alpha_{2}$. The ANCOVA estimator (without controlling for the unobserved confounder $U$; see Equation 2) is given as follows:

$$
\begin{aligned}
\gamma_{\text {ANCOVA }} & =\frac{\operatorname{Cov}\left(Y_{2}, T\right)-\operatorname{Cov}\left(Y_{2}, Y_{1}\right) \operatorname{Cov}\left(T, Y_{1}\right)}{1-\operatorname{Cov}\left(T, Y_{1}\right)^{2}}=\frac{\gamma+\alpha_{2} \alpha_{T}-2 \delta \alpha_{1} \alpha_{T} \gamma-\gamma \delta^{2}-\alpha_{2} \alpha_{T} \alpha_{1}^{2}}{1-2 \delta \alpha_{1} \alpha_{T}-\delta^{2}-\alpha_{1}^{2} \alpha_{T}^{2}} \\
& =\gamma+\frac{\alpha_{2} \alpha_{T}\left(1-\alpha_{1}^{2}\right)}{1-2 \delta \alpha_{1} \alpha_{T}-\delta^{2}-\alpha_{1}^{2} \alpha_{T}^{2}}
\end{aligned} .
$$

Furthermore, the change score estimator is given by:

$$
\begin{aligned}
\gamma_{\text {change }} & =\operatorname{Cov}\left(Y_{2}, T\right)-\operatorname{Cov}\left(Y_{1}, T\right)=\gamma-\delta-\beta \delta+\alpha_{1} \alpha_{2}-\alpha_{1} \alpha_{T}+\beta \alpha_{1} \alpha_{T}+\delta \alpha_{1} \alpha_{2} \\
& =\gamma+\alpha_{T}\left(\alpha_{2}+\beta \alpha_{1}-\alpha_{1}\right)+\delta\left(\beta+\alpha_{1} \alpha_{2}-1\right) .
\end{aligned}
$$

Thus, the change score estimator is unbiased if $\delta=0$ and if $\alpha_{2}+\beta \alpha_{1}=\alpha_{1}$ (i.e., the effect of $U$ is stable with respect to $Y_{1}$ and $Y_{2}$ ).

\section{Inclusion of an Observed Confounder $U$}

It is evident that the ANCOVA estimator is unbiased if the confounder $U$ is included in the regression in Equation 2. However, it can be shown that the change score estimator is biased even if the confounder $U$ is included in the regression in Equation 3:

$$
\begin{aligned}
\gamma_{\text {change }, U} & =\frac{\operatorname{Cov}\left(Y_{2}-Y_{1}, T\right)-\operatorname{Cov}\left(Y_{2}-Y_{1}, U\right) \operatorname{Cov}(T, U)}{1-\operatorname{Cov}(T, U)^{2}}=\frac{\gamma-\delta+\beta \delta-2 \delta \alpha_{1} \alpha_{T} \gamma+\delta \alpha_{1}^{2}-\gamma \alpha_{T}^{2}-\beta \delta \alpha_{1}^{2}-\gamma \delta^{2} \alpha_{1}^{2}}{1-2 \delta \alpha_{1} \alpha_{T}-\alpha_{T}^{2}-\delta^{2} \alpha_{1}^{2}} \\
& =\gamma+\frac{\delta(1-\beta)\left(1-\alpha_{1}^{2}\right)}{1-2 \delta \alpha_{1} \alpha_{T}-\alpha_{T}^{2}-\delta^{2} \alpha_{1}^{2}}
\end{aligned}
$$

In this case, the change score estimator would be unbiased if $\delta=0$ (i.e., past outcome $Y_{1}$ does not affect the treatment).

\section{Bracketing Property}




\section{ANCOVA VERSUS CHANGE SCORE}

We will show that under mild assumptions about the data-generating model (see Figure 1), the ANCOVA and change score estimators fulfill a bracketing property. This means that the two estimators provide upper and lower bounds for the true treatment effect $\gamma$ (Angrist \& Pischke, 2009; Ding \& Li, 2019). First, we assume that the ANCOVA estimator is positively biased (see Equation A1) because it is often reasonable to assume that $\alpha_{1} \alpha_{T}$ is larger than zero. Thus, $\gamma_{\text {Ancova }}$ can be considered to be an upper bound of $\gamma$. Next, we consider the change score estimator and rewrite the bias of $\gamma_{\text {change }}$ as:

$$
\gamma_{\text {change }}=\gamma-\alpha_{T}\left((1-\beta) \alpha_{1}-\alpha_{2}\right)-\delta\left(1-\beta-\alpha_{1} \alpha_{2}\right) \text {. }
$$

We now make the plausible assumption that the cumulative influence of the unobserved confounder $U$ is smaller for the posttest than for the pretest:

$$
(1-\beta) \alpha_{1}-\alpha_{2}>0
$$

Note that the common trend assumption (which is a necessary assumption for the

unbiasedness of $\left.\gamma_{\text {change }}\right)$ is $(1-\beta) \alpha_{1}-\alpha_{2}=0$. Furthermore, given the assumption in Equation A5, it is also plausible to assume that:

$$
1-\beta-\alpha_{1} \alpha_{2}>0
$$

Note that for $\alpha_{1}>0$ this assumption is equivalent to $(1-\beta) \alpha_{1}-\alpha_{1}^{2} \alpha_{2}>0$, which is automatically fulfilled with $\alpha_{1}<1$ and the assumption in Equation A5. Inserting the inequalities of Equations A5 and A6 into Equation A4 shows that it is plausible to expect that the change score estimator is negatively biased and that the following bracketing property holds (in large samples):

$$
\gamma_{\text {change }} \leq \gamma \leq \gamma_{\text {ANCOVA }}
$$

\section{Appendix B: Equivalence to Model with Stable and Transient Components}

The data-generating model that conceptualizes potential confounding influences in terms of stable and transient components of the pretest, posttest, and treatment is given by (see Figure 2; Kenny, 1975; Allison, 1990): 
ANCOVA VERSUS CHANGE SCORE

$$
\begin{aligned}
& Y_{1}=\alpha^{*} U+b_{1} Z_{1} \\
& T=\alpha_{T}^{*} U+\delta^{*} Z_{1}+\varepsilon_{2} \\
& Y_{2}=\alpha^{*} U+b_{2} Z_{2}+\gamma T .
\end{aligned}
$$

As the transient components $Z_{1}$ and $Z_{2}$ are allowed to be correlated, we can write $Z_{2}=r_{Z_{1} Z_{2}} Z_{2}$ $+\varepsilon_{3}$. If we now set $\beta^{*}=b_{2} r_{Z_{1} Z_{2}}$, the equation for $Y_{2}$ can be modified to $Y_{2}=\alpha^{*} U+\beta^{*} Z_{1}+\gamma T+$ $\varepsilon_{3}$. We now reorder the terms in the Equation for $T$ as follows:

$$
\begin{aligned}
T & =\alpha_{T}^{*} U+\delta^{*} Z_{1}+\varepsilon_{2}=\alpha_{T}^{*} U+\delta^{*}\left(Y_{1}-\alpha^{*} U\right) / b_{1}+\varepsilon_{2} \\
& =\left(\alpha_{T}^{*}-\delta^{*} \alpha^{*} / b_{1}\right) U+\left(\delta^{*} / b_{1}\right) Y_{1}+\varepsilon_{2} \\
& =\alpha_{1} U+\delta_{1} Y_{1}+\varepsilon_{2}
\end{aligned}
$$

by using appropriate definitions of $\alpha_{1}=\alpha_{T}^{*}-\delta^{*} \alpha^{*} / b_{1}$ and $\delta=\delta^{*} / b_{1}$. Similarly,

$$
\begin{aligned}
Y_{2} & =\alpha^{*} U+\beta^{*} Z_{1}+\gamma T+\varepsilon_{3}=\alpha^{*} U+\frac{\beta^{*}\left(Y_{1}-\alpha^{*} U\right)}{b_{1}}+\gamma T+\varepsilon_{3}= \\
& =\alpha^{*}\left(1-\frac{\beta^{*}}{b_{1}}\right) U+\left(\frac{\beta^{*}}{b_{1}}\right) Y_{1}+\gamma T+\varepsilon_{3} \\
& =\alpha_{2} U+\beta Y_{1}+\gamma T+\varepsilon_{3}
\end{aligned}
$$

using $\alpha_{2}=\alpha^{*}\left(1-\frac{\beta^{*}}{b_{1}}\right)$ and $\beta=\left(\frac{\beta^{*}}{b_{1}}\right)$. Thus, the model with transient and stable components (Equation B1 and Figure 2) can be considered to be a special case of the data-generating model with an unobserved confounder in the main text (see Figure 1). It should be emphasized that in the model of Kenny (1975) and Allison (1990), the existence of a confounder with a stable effect is represented by assuming the same coefficient for the effect of $U$ on $Y_{1}$ and $Y_{2}\left(\alpha_{1}=\alpha_{2}\right)$.

\section{Appendix C: Bias for the ANCOVA and Change Score Approaches under Measurement Error}

In this appendix, we sketch the bias derivations for the ANCOVA and change score approaches under measurement error. Assuming that the data-generating model in Equation 8 is true (see Figure 3), the covariances between the observed variables $\tilde{Y}_{1}, \tilde{Y}_{2}$, and $\tilde{T}$ are derived 


\section{ANCOVA VERSUS CHANGE SCORE}

as follows: $\operatorname{Cov}\left(\widetilde{T}, \tilde{Y}_{1}\right)=\delta+\alpha_{1} \alpha_{T} ; \operatorname{Cov}\left(\tilde{Y}_{2}, \tilde{Y}_{1}\right)=\beta+\delta \gamma+\alpha_{1} \alpha_{2}+\alpha_{1} \alpha_{T} \gamma+\rho_{E} ; \operatorname{Cov}\left(\tilde{Y}_{2}, \tilde{T}\right)=$ $\gamma+\beta \delta+\alpha_{2} \alpha_{T}+\beta \alpha_{1} \alpha_{T}+\delta \alpha_{1} \alpha_{2}$. For the ANCOVA estimator (see Equation 2), we specify a measurement error correction for $\tilde{Y}_{1}$ and $\tilde{T}$, where $\lambda_{1}$ and $\lambda_{2}$ are the ratios of the estimated and the true reliabilities, that is, $\lambda_{1}=\hat{r}_{1} / r_{1}$ and $\lambda_{2}=\hat{r}_{2} / r_{2}$. The bias of the corrected ANCOVA estimator is then given as follows:

$$
\begin{aligned}
& \gamma_{\text {ANCOVA }}^{*}=\frac{\lambda_{1} \operatorname{Cov}\left(\tilde{Y}_{2}, \tilde{T}\right)-\operatorname{Cov}\left(\tilde{Y}_{2}, \tilde{Y}_{1}\right) \operatorname{Cov}\left(\tilde{T}, \tilde{Y}_{1}\right)}{\lambda_{1} \lambda_{2}-\operatorname{Cov}\left(\widetilde{T}, \tilde{Y}_{1}\right)^{2}} \\
& =\frac{\lambda_{1} \gamma-\beta \delta-\delta \rho_{E}+\beta \delta \lambda_{1}+\alpha_{2} \alpha_{T} \lambda_{1}-\beta \alpha_{1} \alpha_{T}-\delta \alpha_{1} \alpha_{2}-\alpha_{1} \alpha_{T} \rho_{E}+\beta \alpha_{1} \alpha_{T} \lambda_{1}+\delta \alpha_{1} \alpha_{2} \lambda_{1}-2 \delta \alpha_{1} \alpha_{2} \gamma-\gamma \delta^{2}-\alpha_{2} \alpha_{T} \alpha_{1}^{2}-\gamma \alpha_{1}^{2} \alpha_{T}^{2}}{\lambda_{1} \lambda_{2}-2 \delta \alpha_{1} \alpha_{T}-\delta^{2}-\alpha_{1}^{2} \alpha_{T}^{2}} \\
& =\gamma+\frac{\alpha_{2} \alpha_{T}\left(\lambda_{1}-\alpha_{1}^{2}\right)+\left(\lambda_{1}-1\right)\left(\beta \delta+\delta \alpha_{1} \alpha_{2}+\beta \alpha_{1} \alpha_{T}\right)-\left(\lambda_{2}-1\right) \lambda_{1} \gamma-\rho_{E}\left(\alpha_{1} \alpha_{T}+\delta\right)}{\lambda_{1} \lambda_{2}-2 \delta \alpha_{1} \alpha_{T}-\delta^{2}-\alpha_{1}^{2} \alpha_{T}^{2}}
\end{aligned}
$$

The bias of the change score estimator that is corrected for measurement in $\tilde{T}$ is given by:

$$
\begin{aligned}
\gamma_{\text {change }}^{*} & =\frac{\operatorname{Cov}\left(\tilde{Y}_{2}, \tilde{T}\right)-\operatorname{Cov}\left(\tilde{Y}_{1}, \tilde{T}\right)}{\lambda_{2}} \\
& =\frac{1}{\lambda_{2}}\left[\gamma-\alpha_{T}\left((1-\beta) \alpha_{1}-\alpha_{2}\right)-\delta\left(1-\beta-\alpha_{1} \alpha_{2}\right)\right] \\
& =\gamma+\frac{\lambda_{2}-1}{\lambda_{2}} \gamma+\frac{1}{\lambda_{2}}\left[\alpha_{T}\left(\alpha_{2}+\beta \alpha_{2}-\alpha_{1}\right)+\delta\left(\beta+\alpha_{1} \alpha_{2}-1\right)\right]
\end{aligned}
$$

